\title{
Formação profissional de enfermeiro gerontológico: revisão integrativa
}

\author{
Professional training of gerontological nurses: integrative review \\ Formación profesional de enfermeras gerontológicas: revisión integradora
}

Recebido: 24/08/2021 | Revisado: 29/08/2021 | Aceito: 02/09/2021 | Publicado: 05/09/2021

\author{
Miriam Marinho Chrizostimo \\ ORCID: https://orcid.org/0000-0001-7598-4637 \\ Universidade Federal Fluminense, Brasil \\ E-mail: miriammarinho@hotmail.com \\ Célia Pereira Caldas \\ ORCID: https://orcid.org/0000-0001-6903-1778 \\ Universidade do Estado do Rio de Janeiro, Brasil \\ E-mail: celpcaldas@hotmail.com
}

\begin{abstract}
Resumo
Objetivo da pesquisa foi analisar a formação profissional na área da gerontologia, por meio das produções científicas para o reconhecimento das estratégias de ensino utilizados na educação em enfermagem geriátrica, tanto para a qualidade do cuidado do enfermeiro em relação à família como o de cuidadores familiares. O método utilizado foi a Revisão Integrativa da Literatura qualitativa realizada de julho a agosto de 2020. Através da combinação de operadores booleanos "and" e "or", identificou-se cinquenta produções científicas, porém, oito atendiam os requisitos do estudo tendo em vista os critérios de inclusão/exclusão. No ensino da gerontologia, a formação dos estudantes de enfermagem deve ser fortalecida por métodos e estratégias de aprendizagem voltados para o desenvolvimento do conjunto de competências para o cuidado. Entretanto, a educação em enfermagem está relacionada com a extensão de resultados significativos da formação profissional, o processo de cuidar da pessoa idosa e a qualidade do cuidado familiar.
\end{abstract}

Palavras-chave: Ensino; Educação em enfermagem; Enfermagem; Estudantes de enfermagem; Aprendizagem.

\begin{abstract}
The objective of the research was to analyze professional training in the field of gerontology, through scientific productions for the recognition of teaching strategies used in geriatric nursing education, both for the quality of care provided by nurses in relation to the family and that of family caregivers. The method used was the Integrative Review of Qualitative Literature held from July to August 2020. Through the combination of Boolean operators "and" and "or", fifty scientific productions were identified, however, eight met the requirements of the study in view of the inclusion/exclusion criteria. In the teaching of gerontology, the training of nursing students must be strengthened by methods and learning strategies aimed at developing a set of skills for care. However, nursing education is related to the extension of significant results of professional training, the process of caring for the elderly and the quality of family care.
\end{abstract}

Keywords: Teaching; Education; Nursing; Nursing students; Learning.

\section{Resumen}

El objetivo de la investigación fue analizar la formación profesional en el campo de la gerontología, a través de producciones científicas para el reconocimiento de las estrategias docentes empleadas en la formación en enfermería geriátrica, tanto para la calidad de los cuidados que brindan las enfermeras en relación con la familia como la de los cuidadores familiares. El método utilizado fue la Revisión Integrativa de Literatura Cualitativa realizada de julio a agosto de 2020. Mediante la combinación de los operadores booleanos "y" y "o", se identificaron cincuenta producciones científicas, sin embargo, ocho cumplieron con los requisitos del estudio en vista de la criterios de inclusión / exclusión. En la enseñanza de la gerontología, la formación de los estudiantes de enfermería debe fortalecerse con métodos y estrategias de aprendizaje dirigidas a desarrollar un conjunto de habilidades para el cuidado. Sin embargo, la formación en enfermería está relacionada con la extensión de resultados significativos de la formación profesional, el proceso de cuidado del anciano y la calidad del cuidado familiar.

Palabras clave: Enseñanza; Educación en enfermería; Enfermería; Estudiantes de enfermería; Aprendizaje.

\section{Introdução}

A formação do enfermeiro em gerontologia revela a construção do processo ensino-aprendizagem, por meio das instituições que formam este profissional. Há de se considerar ainda, que este processo ocorre em espaços além das Instituições 
de Ensino Superior (IES), tendo em vista a relação entre as educações formal, não formal ou informal, que colaboram para a formação do ator social promotor da ação do cuidado a pessoa idosa, que neste estudo é o enfermeiro.

Nesse contexto, realizou-se uma revisão integrativa sobre a formação profissional do enfermeiro em gerontologia. Este tema vai ao encontro das linhas de pesquisa sobre cuidado familiar e formação profissional, estando inter-relacionadas com as áreas de tecnologia; gestão; educação; ensino e saúde; contexto histórico, social e político, abarcando as ideias contidas nas publicações dos Grupos de Pesquisa Enfermagem em Saúde do Idoso da Universidade do Estado do Rio de Janeiro (GEPESI/UERJ) e Gestão da Formação e Qualificação Profissional: Educação e Saúde da Universidade Federal Fluminense (GESPRO/UFF).

A investigação relacionada à formação profissional em gerontologia se origina da inquietação derivada da experiência docente. A formação do enfermeiro precisa ser constituída de modo transversal, para atender a demanda ora existente da população brasileira.

No Brasil, existem mais de 28 milhões de pessoas acima dos 60 anos, número que representa $13 \%$ da população do país, sendo esse percentual projetado para dobrar até 2047 (Instituto Brasileiro de Geografia e Estatística, 2018). Com isso, se faz necessário o aprimoramento da formação do enfermeiro, pois as características da população idosa são singulares e as especificidades do cuidar dessas pessoas precisam ser trabalhadas como competências e habilidades profissionais.

Nesse sentido, na graduação em enfermagem o campo da educação precisa se articular com o campo da saúde como prática social. Quando formação do enfermeiro no curso de graduação é estabelecida com base na compreensão da saúde como prática social, este profissional terá clareza sobre o seu papel na sociedade no atendimento aos anseios e necessidades da pessoa idosa e de seus cuidadores familiares dentro de um sistema de relações objetivas, o que traz para a IES a responsabilidade social do cuidar.

Um sistema de relações objetivas envolve o respeito à cultura e ao conhecimento adquirido pela pessoa idosa na sua experiência de vida. Por isso, espera-se que a formação obtida nos cursos de graduação valorize o conhecimento da pessoa idosa. Este conhecimento deve ser considerado como o cerne do cuidado. Assim, desvela-se a importância da conscientização em relação ao ensino crítico-reflexivo, com compromisso social e humanizado, o qual colabora para a formação de profissionais aptos para atenderem às demandas do indivíduo, família e sociedade.

A Política Nacional da Pessoa Idosa, Portaria no 2.528 de 19 de outubro de 2006 (Brasil, 2006), traz à luz o direito universal e integral à saúde da pessoa idosa conquistado pela sociedade na Constituição de 1988 (Brasil, 1988) e reforça a importância do Sistema Único de Saúde (SUS), criado por meio da Lei Orgânica da Saúde no 8.080/90 (Brasil, 1990). Neste sentido, a saúde e a dignidade das pessoas idosas devem ser a meta da formação profissional do enfermeiro. Destaca-se que esta formação deve, principalmente, respeitar a singularidade e a especificidade do cuidado para aquela parcela da população que teve, por uma série de razões, o processo de envelhecimento marcado por agravos que impuseram limitações ao seu bemestar (Brasil, 2006). Com esse pensamento, pode-se ressaltar o seguinte questionamento: Quais são as estratégias de ensino utilizados na educação em enfermagem geriátrica?

A pesquisa tem o objetivo de analisar a formação profissional na área da gerontologia, por meio das produções científicas para o reconhecimento das estratégias de ensino utilizados na educação em enfermagem geriátrica, tanto para a qualidade do cuidado do enfermeiro em relação à família como o de cuidadores familiares.

\section{Metodologia}

Trata-se de uma revisão integrativa da literatura que seguiu as recomendações do protocolo PRISMA (Statement for Reporting Systematic Reviews and Meta-analyses of Studies) de forma adaptada, elaborada nos meses de julho e agosto de 2020. A sua realização seguiu as seguintes etapas: 1) Identificação do tema e formulação da questão norteadora; 2) 
Estabelecimento dos critérios para inclusão e exclusão dos estudos; 3) Definição das bases de dados da pesquisa; 4) Determinação dos recursos da busca bibliográfica; 5) Definição das informações a serem extraídas dos estudos selecionados; 6) Avaliação dos estudos incluídos; 7) Interpretação dos resultados; e 8) Apresentação da revisão (Mendes, Silveira \& Galvão, 2008).

Foram escolhidas as seguintes bases de dados para a realização da pesquisa: Sistema Online de Busca e Análise de Literatura Médica - Medical Literature Analysis and Retrieval System Online (MEDLINE); Literatura Científica e Técnica da América Latina e Caribe (LILACS); Base de Dados Bibliográfica especializada na área de Enfermagem (BDENF).

A busca bibliográfica foi estabelecida pelo uso dos descritores de maneira particular, com a determinação dos seguintes filtros: 1. Texto completo; 2. Base de dados (MEDLINE, LILACS e BDENF); 3. Assunto geral (geriatria; ensino; currículo; envelhecimento; estudante de enfermagem; enfermagem geriátrica; e competência profissional); 4. Tipos de estudo todos; 5. Idiomas (inglês; português; espanhol e francês); 6. Intervalo de publicação - últimos 5 anos (2015-2020).

Em seguida, foram estabelecidos os critérios de inclusão e exclusão dos estudos. Os critérios de inclusão foram: artigos científicos escritos nos idiomas português, francês, espanhol e inglês publicados entre os anos de 2015 e 2020, indexados com textos completos, disponíveis online e que tivessem a abordagem do tema em caráter nacional. E, os de exclusão: artigos de revisão e/ou pesquisa bibliográfica de diferentes tipos, recuperados de maneira repetida nas diferentes bases de dados.

Foram definidas as bases de dados da pesquisa, por meio do Portal da Biblioteca Virtual em Saúde (BVS), ao considerar a necessidade de obter dados nacionais e internacionais. Os termos foram escolhidos nos idiomas inglês, espanhol e seus respectivos sinônimos em português (Descritor em Ciência da Saúde, 2016): 1. Nurses; enfermeras y enfermeros; enfermeira, com sinônimos de enfermeira; de enfermeira e enfermeiro; de enfermeiro e enfermeira; de enfermeiros registrados; de enfermeiro e enfermeiras; 2. Teaching; enseñanza; ensino, sendo docência e pedagogia; 3. Students, nursing; estudiantes de enfermería; estudantes de enfermagem, tendo como sinônimo enfermeiras estudantes, alunos de Enfermagem e enfermeiros estudantes; 4. Education, nursing; educación en enfermería; educação em enfermagem com sinônimo de curso de enfermagem e cursos de enfermagem, para esta pesquisa desconsiderou-se a sinonímia curso de assistente em enfermagem pediátrica, por estar em desacordo com o objeto do estudo; 5. Learning; aprendizaje; aprendizagem como aprendizado, sem considerar a fenomenografia, pois é um método de pesquisa qualitativa pouco utilizado (Cherman \& Rocha-Pinto, 2016). Ele visa capturar diversas concepções dos indivíduos acerca de um fenômeno, isto é, modos qualitativamente diferentes de experimentá-lo e concebê-lo (Marton, 1981; Marton \& Booth, 1997; Sandberg, 2000).

As definições dos descritores em português consideradas neste estudo foram: 1. Enfermeiras e enfermeiros, profissionais graduados em escola acreditada de enfermagem e que passaram pelo exame de licenciamento nacional para praticar enfermagem. Elas/eles prestam serviços à pacientes que requerem assistência para recuperar ou manter sua saúde física ou mental; 2. Ensino é o processo formal e organizado de transmissão de conhecimento para uma pessoa ou um grupo; 3. Estudantes de enfermagem são os indivíduos matriculados em uma escola de enfermagem ou em um programa de educação formal que leva à graduação em enfermagem; 4. Educação em enfermagem, através da utilização de artigos em geral que dizem respeito a educação em enfermagem. 5. Aprendizagem como mudança de comportamento relativamente duradoura que resulta da experiência passada ou da prática. Esse conceito inclui a aquisição de conhecimento (Descritor em Ciência da Saúde, 2016).

Torna-se necessário esclarecer que não houve a utilização de termos genéricos, como: 1. Gerontologia - ramo da medicina voltado para os aspectos fisiológicos e patológicos dos idosos, inclusive dos problemas clínicos do envelhecimento e da senilidade; 2. Formação profissional - treinar pessoa ou grupo de pessoas no conhecimento ou na aplicação prática e teórica de uma determinada atividade, que no caso desta pesquisa é inadequado, pois se espera a transformação no ato de cuidar para a 
prática com crítica e reflexão (Descritor em Ciência da Saúde, 2016). Sugere-se a introdução da palavra gerontológica no Descritor em Ciência da Saúde (DeCS), tendo em vista a evolução deste termo na prática da enfermagem e por se configurar em uma variação da palavra gerontologia, indicada pelo ramo da lexicologia, chamado de onomasiologia. Essa é uma área da linguística que estuda os significados (abstrato ou concreto) dos termos a partir de um contexto real (Babini, 2006).

A primeira pesquisa foi realizada em 04 de julho de 2020, sem cruzamentos entre os descritores o que resultou no total de 3.558.975 artigos científicos, distribuídos entre os seguintes termos: Ensino (457.499), Gerontologia (17.538), Formação profissional (26.600), Enfermeira (122.806) e Pessoa idosa (3.057.938).

A continuidade da pesquisa em 07 de julho de 2020 foi feita com a busca dos textos com a utilização dos operadores booleanos "and" e "or" através de oito combinações, destacadas a seguir: tw:((mh:(enfermeira)) AND (mh:(ensino))) AND (fulltext:("1") AND db:("MEDLINE" OR "BDENF" OR "LILACS") AND mj:("Enfermeiras e Enfermeiros" OR "Ensino" OR "Estudantes de Enfermagem" OR "Educação em Enfermagem" OR "Bacharelado em Enfermagem" OR "Aprendizagem") AND la:("en" OR "pt" OR "es")) AND (year_cluster:[2015 TO 2020]). Como resultado, identificou-se 50 produções científicas, excluindo-se 42 , tendo em vista o não atendimento aos critérios de inclusão/exclusão do estudo. Com a realização dessa estratégia de busca, foram selecionadas 8 produções científicas em inglês, que atendiam os requisitos do estudo, conforme apresentado no Quadro 1.

Quadro 1 - Estratégia de busca da quarta pesquisa com os critérios do estudo, Niterói, RJ, Brasil.

\begin{tabular}{|c|c|c|c|}
\hline \multicolumn{4}{|c|}{$\mathbf{N}^{0}$ DE PRODUÇÕES CIENTÍFICAS $(\mathrm{PC})=50^{*}$} \\
\hline Critérios & Descrição & $\begin{array}{l}\text { Número } \\
\text { de Artigos }\end{array}$ & $\begin{array}{c}\text { Total } \\
\text { Selecionado }\end{array}$ \\
\hline \multirow{3}{*}{ INCLUSÃO } & $\begin{array}{l}\text { Artigos científicos/idiomas português, francês, espanhol e } \\
\text { inglês }\end{array}$ & 50 & \multirow{6}{*}{8} \\
\hline & $\begin{array}{c}\text { Produções científicas indexadas/ textos completos e } \\
\text { disponíveis online }\end{array}$ & 18 & \\
\hline & Caráter nacional/internacional como objeto & 18 & \\
\hline \multirow{3}{*}{ EXCLUSÃO } & Itens contrários a inclusão & 30 & \\
\hline & Artigos de revisão/pesquisa bibliográfica/ diferentes tipos & 10 & \\
\hline & $\begin{array}{l}\text { Documentos recuperados de maneira repetida/diferentes bases } \\
\text { de dados }\end{array}$ & 2 & \\
\hline
\end{tabular}

* Estratégias de busca: tw:((mh:(enfermeira)) AND (mh:(ensino))) AND (fulltext:("1") AND db:("MEDLINE" OR "BDENF" OR "LILACS") AND mj:("Enfermeiras e Enfermeiros" OR "Ensino" OR "Estudantes de Enfermagem" OR "Educação em Enfermagem" OR "Bacharelado em Enfermagem" OR "Aprendizagem") AND la:("en" OR "pt" OR "es")) AND (year_cluster:[2015 TO 2020]) = 50. Fonte: Levantamento de dados.

Ressalta-se que o presente artigo está em consonância com a resolução 466 de 2012 e dispensa aprovação no Comitê de Ética em Pesquisa por se tratar de estudo de Revisão.

De acordo com os pressupostos de Bourdieu (2007, 2011), a limitação do estudo se expressa nas experiências internacionais, pois qualquer tentativa de internacionalização social através da educação pressupõe a necessidade de trabalho contínuo de denúncia, crítica e a transcendência do "discurso" ideológico que se incorpora ao "discurso" pedagógico. Sendo assim, a formação do enfermeiro gerontológico deve "beber" na fonte de cada espaço social, visto que se vive num país que necessita de contextualização da própria cultura. 


\section{Resultados}

O Quadro 2 revela as informações extraídas dos oito estudos selecionados: item para a identificação das produções científicas, chamadas de PC1, PC2, assim por diante até PC8; título; o ano de publicação; id:biblio, que é a identificação; bem como, os temas abordados em português que se encontram nos artigos e o(s) objetivo(s) dos mesmos.

Quadro 2 - Informações das produções científicas (base de dados MEDLINE), Niterói, RJ, Brasil.

\begin{tabular}{|c|c|c|c|c|c|}
\hline Item & $\begin{array}{c}\text { Título ou } \\
\text { referência(s) }\end{array}$ & Ano & Temas Abordados & Objetivo(s) & Id: Biblio \\
\hline PC1 & $\begin{array}{l}\text { Desenvolvimento de } \\
\text { vinhetas para } \\
\text { aprendizagem e } \\
\text { desenvolvimento } \\
\text { profissional }\end{array}$ & 2018 & $\begin{array}{c}\text { Educação em Enfermagem, Enfermagem } \\
\text { Geriátrica/educação, Geriatria/educação, } \\
\text { Materiais de Ensino, Ensino, Competência } \\
\text { Clínica, Educação, Educação em } \\
\text { Enfermagem/métodos, Educação em } \\
\text { Enfermagem/normas, Humanos, Motivação, } \\
\text { Melhoria de qualidade, Ensino/psicologia, } \\
\text { Ensino/normas }\end{array}$ & $\begin{array}{l}\text { Desenvolver vinhetas de } \\
\text { tarefas de trabalho como } \\
\text { gatilho para processos de } \\
\text { aprendizagem e } \\
\text { desenvolvimento profissional } \\
\text { do enfermeiro geriátrico. }\end{array}$ & $\begin{array}{c}\text { mdl- } \\
27732525\end{array}$ \\
\hline PC2 & $\begin{array}{l}\text { Trazendo um romance } \\
\text { para a prática: um } \\
\text { estudo interpretativo da } \\
\text { leitura de um romance } \\
\text { em um curso de estágio } \\
\text { de graduação em } \\
\text { enfermagem }\end{array}$ & 2017 & $\begin{array}{c}\text { Livros, Bacharelado em } \\
\text { Enfermagem/métodos, Leitura, } \\
\text { Estudantes de Enfermagem/psicologia, } \\
\text { Ensino/tendências, Atitude do Pessoal de } \\
\text { Saúde, Currículo/normas, } \\
\text { Currículo/tendências, Grupos Focais, } \\
\text { Geriatria/tendências, Humanos, Pesquisa } \\
\text { Qualitativa, Universidades/organização \& } \\
\text { administração, Recursos Humanos }\end{array}$ & $\begin{array}{l}\text { Ampliar a compreensão sobre os } \\
\text { significados que os participantes } \\
\text { da pesquisa atribuíram ao } \\
\text { exercício da leitura do romance, } \\
\text { com relação ao desenvolvimento } \\
\text { enquanto estudantes de } \\
\text { enfermagem, por meio da } \\
\text { abordagem hermenêutica, que } \\
\text { usa o diálogo ao longo do estudo } \\
\text { para conhecer as perspectivas } \\
\text { dos participantes e da equipe de } \\
\text { pesquisa interdisciplinar. }\end{array}$ & $\begin{array}{c}\text { mdl- } \\
28432924\end{array}$ \\
\hline PC3 & $\begin{array}{l}\text { Cooperativa } \\
\text { trabalhando no cuidado } \\
\text { de idosos: Projeto de } \\
\text { pesquisa e ensino da } \\
\text { Cooperative for } \\
\text { Healthy Aging }\end{array}$ & 2016 & $\begin{array}{c}\text { Pesquisa Biomédica, Comportamento } \\
\text { Cooperativo, Prestação Integrada de } \\
\text { Cuidados de Saúde/organização \& } \\
\text { administração, Geriatria/educação, Pesquisa } \\
\text { sobre Serviços de Saúde, Serviços de Saúde } \\
\text { para Idosos/organização \& administração, } \\
\text { Relações Interinstitucionais, Parcerias } \\
\text { Público-Privadas, Ensino, Mobilidade } \\
\text { Ocupacional, Difusão de Inovações, Mão de } \\
\text { Obra em Saúde, Humanos, Entrevistas como } \\
\text { Assunto, New South Wales, Inovação } \\
\text { Organizacional, Objetivos Organizacionais, } \\
\text { Desenvolvimento de Programas. }\end{array}$ & $\begin{array}{l}\text { Descrever os mecanismos de } \\
\text { parceria que apoiaram o ensino } \\
\text { e a investigação no cuidado do } \\
\text { idoso, num dos } 16 \text { projetos } \\
\text { financiados no âmbito do } \\
\text { projeto serviço de ensino e } \\
\text { investigação no cuidado ao } \\
\text { idoso. }\end{array}$ & $\begin{array}{c}\text { mdl- } \\
27225980\end{array}$ \\
\hline PC4 & $\begin{array}{l}\text { Atendimento } \\
\text { interprofissional de } \\
\text { idosos: utilizando o } \\
\text { ambiente virtual de } \\
\text { aprendizagem }\end{array}$ & 2016 & $\begin{array}{c}\text { Comportamento Cooperativo, Educação } \\
\text { Continuada/métodos, Geriatria/educação, } \\
\text { Relações Interprofissionais, } \\
\text { Aprendizagem, Ensino, Competência } \\
\text { Clínica/normas, humanos. }\end{array}$ & $\begin{array}{l}\text { Examinar as percepções dos } \\
\text { alunos sobre o ambiente virtual } \\
\text { de aprendizagem que foi } \\
\text { desenvolvido para melhorar as } \\
\text { competências interprofissionais. }\end{array}$ & $\begin{array}{c}\text { mdl- } \\
27166735\end{array}$ \\
\hline PC5 & $\begin{array}{l}\text { Caminhos para o } \\
\text { avanço da pesquisa } \\
\text { relevante para políticas } \\
\text { de envelhecimento em } \\
\text { ambientes acadêmicos }\end{array}$ & 2016 & $\begin{array}{c}\text { Envelhecimento , Pesquisa Biomédica, } \\
\text { Geriatria, Política de Saúde/tendências, } \\
\text { Pesquisa Médica Translacional, } \\
\text { Envelhecimento/fisiologia, } \\
\text { Envelhecimento/psicologia, Pesquisa } \\
\text { Biomédica/educação, Pesquisa } \\
\text { Biomédica/organização \& administração, } \\
\text { Diversidade Cultural, Docentes de } \\
\text { Medicina/normas, Grupos Focais, } \\
\text { Geriatria/educação, Geriatria/tendências, } \\
\text { Humanos, Formulação de Políticas, }\end{array}$ & $\begin{array}{l}\text { Examinar barreiras e caminhos } \\
\text { para desenvolvimento e } \\
\text { manutenção de uma agenda de } \\
\text { pesquisa relevante para } \\
\text { políticas de envelhecimento } \\
\text { em ambientes acadêmicos, } \\
\text { como também, os desafios } \\
\text { adicionais associados à } \\
\text { participação em grupos } \\
\text { minoritários nessa busca. }\end{array}$ & $\begin{array}{c}\text { mdl- } \\
26849290\end{array}$ \\
\hline
\end{tabular}




\begin{tabular}{|c|c|c|c|c|c|}
\hline & & & $\begin{array}{c}\text { Ensino/organização \& administração, } \\
\text { Ensino/normas, Pesquisa Médica } \\
\text { Translacional/educação, Pesquisa Médica } \\
\text { Translacional/organização \& administração }\end{array}$ & & \\
\hline PC6 & $\begin{array}{l}\text { Desenvolvimento de } \\
\text { um currículo para } \\
\text { profissionais de } \\
\text { enfermagem avançados } \\
\text { que trabalham com } \\
\text { idosos com fragilidade } \\
\text { no hospital de urgência } \\
\text { por meio de um } \\
\text { processo Delphi } \\
\text { modificado }\end{array}$ & 2016 & \begin{tabular}{|} 
Técnica Delfhi, Educação de Pós-Graduação \\
em Enfermagem/métodos, Idoso Fragilizado, \\
Geriatria/educação, Hospitalização, \\
Profissionais de Enfermagem/educação, \\
Ensino/métodos, Fatores Etários, Idoso, \\
Competência Clínica, Consenso, Currículo, \\
Educação de Pós-Graduação em \\
Enfermagem/normas, Geriatria/normas, \\
Guias como Assunto, Humanos, \\
Profissionais de Enfermagem/normas, Papel \\
do Profissional de Enfermagem, \\
Desenvolvimento de Programas, \\
Ensino/normas.
\end{tabular} & $\begin{array}{l}\text { Estabelecer consenso dos } \\
\text { especialistas sobre a descrição } \\
\text { de papéis e competências } \\
\text { essenciais para os Profissionais } \\
\text { de Enfermagem Avançados } \\
\text { (ANPs) que trabalham com } \\
\text { idosos com fragilidade para } \\
\text { desenvolver um currículo. }\end{array}$ & $\begin{array}{c}\text { Idl- } \\
26764394\end{array}$ \\
\hline PC7 & $\begin{array}{l}\text { Aprendizagem } \\
\text { facilitada para o } \\
\text { avanço da geriatria: } \\
\text { aumentando a } \\
\text { capacidade do corpo } \\
\text { docente de } \\
\text { enfermagem de ensinar } \\
\text { aos alunos como cuidar } \\
\text { de adultos mais velhos }\end{array}$ & 2015 & $\begin{array}{c}\text { Educação Continuada em } \\
\text { Enfermagem/organização \& } \\
\text { administração, Docentes de Enfermagem, } \\
\text { Enfermagem Geriátrica/educação, } \\
\text { Geriatria/educação, Desenvolvimento de } \\
\text { Pessoal/organização \& administração, } \\
\text { Ensino/métodos, Adulto, Atitude do } \\
\text { Pessoal de Saúde, Humanos, Masculino, } \\
\text { Mentores, Pessoa de Meia-Idade, } \\
\text { Avaliação de Programas e Projetos de } \\
\text { Saúde }\end{array}$ & $\begin{array}{l}\text { O programa Aprendizagem } \\
\text { Facilitada para o Avanço da } \\
\text { Geriatria (FLAG) foi projetado } \\
\text { para aumentar o número de } \\
\text { professores de enfermagem em } \\
\text { programas de pré-licenciamento } \\
\text { com conhecimento básico sobre } \\
\text { envelhecimento e eficácia de } \\
\text { ensino para preparar os alunos } \\
\text { para prestar cuidados seguros e } \\
\text { de alta qualidade para adultos } \\
\text { mais velhos. }\end{array}$ & $\begin{array}{l}\text { ID: mdl- } \\
26055777\end{array}$ \\
\hline PC8 & $\begin{array}{l}\text { A viabilidade do } \\
\text { etnodrama como } \\
\text { intervenção para } \\
\text { destacar o potencial da } \\
\text { vida adulta para } \\
\text { estudantes de } \\
\text { enfermagem e idosos }\end{array}$ & 2015 & $\begin{array}{c}\text { Geriatria, Relações Enfermeiro- } \\
\text { Paciente/ética, Psicodrama, Estudantes de } \\
\text { Enfermagem/psicologia, Adulto, Idoso, } \\
\text { Geriatria/educação, Geriatria/métodos, } \\
\text { Humanos, Relação entre Gerações, } \\
\text { Masculino, Avaliação de Programas e } \\
\text { Projetos de Saúde, Psicodrama/educação, } \\
\text { Psicodrama/métodos, Ensino }\end{array}$ & $\begin{array}{l}\text { Avaliar a viabilidade do uso do } \\
\text { etnodrama como uma } \\
\text { intervenção para destacar o } \\
\text { potencial na velhice. }\end{array}$ & $\begin{array}{l}\text { ID: mdl- } \\
25671588\end{array}$ \\
\hline
\end{tabular}

Fonte: Levantamento de dados.

A coleta de dados que foi realizada pelo fichamento favoreceu o refinamento dos achados da pesquisa. No Quadro 3, demonstra-se os elementos, a centralidade da pesquisa e a metodologia dessas pesquisas.

Quadro 3 - Apresentação dos métodos, resultados e a centralidade da pesquisa, Niterói, RJ, Brasil.

\begin{tabular}{|c|l|l|l|}
\hline Item & \multicolumn{1}{|c|}{ Elemento da pesquisa } & \multicolumn{1}{c|}{ Centro da pesquisa } & \multicolumn{1}{c|}{ Metodologia } \\
\hline PC1 & $\begin{array}{l}\text { Análise de como as vinhetas devem } \\
\text { ser elaboradas, ao considerar o seu } \\
\text { uso como gatilho para o processo de } \\
\text { aprendizagem e o desenvolvimento } \\
\text { profissional. Com isso, os autores } \\
\text { elaboraram quatro vinhetas, nas quais } \\
\text { possuem a descrição das tarefas de } \\
\text { trabalho da equipe de enfermagem } \\
\text { geriátrica. }\end{array}$ & $\begin{array}{l}\text { Como as vinhetas devem ser } \\
\text { projetadas, para usá-las como gatilho } \\
\text { para o processo de aprendizagem e o } \\
\text { desenvolvimento profissional. }\end{array}$ & $\begin{array}{l}\text { Os autores da pesquisa analisaram os dados } \\
\text { coletados, assim, emergiram os requisitos } \\
\text { necessários para o desenvolvimento de quatro } \\
\text { vinhetas. Estas vinhetas foram validadas em } \\
\text { estudo que contou com 12 entrevistados } \\
\text { especialistas, que possuem domínio de } \\
\text { conhecimento na área da enfermagem } \\
\text { geriátrica. }\end{array}$ \\
\hline $\begin{array}{l}\text { PC2 } \\
\text { atribuídos pelos participantes da } \\
\text { pesquisa, com relação ao } \\
\text { desenvolvimento dos estudantes de } \\
\text { enfermagem por meio do exercício } \\
\text { da leitura do romance. }\end{array}$ & $\begin{array}{l}\text { Os significados que os participantes } \\
\text { da pesquisa atribuem ao exercício da } \\
\text { leitura romance para o } \\
\text { desenvolvimento dos estudantes de } \\
\text { enfermagem. }\end{array}$ & $\begin{array}{l}\text { Estudo interpretativo onde os instrutores de } \\
\text { enfermagem clínica utilizaram como } \\
\text { instrumento do estudo um romance com os } \\
\text { estagiários do curso de graduação. A pesquisa } \\
\text { foi realizada pela equipe interdisciplinar, com } \\
\text { inclusão de enfermeiras, um médico e um } \\
\text { instrutor de inglês. }\end{array}$ \\
\hline
\end{tabular}




\begin{tabular}{|c|c|c|c|}
\hline PC3 & $\begin{array}{l}\text { Melhoraria na capacidade de cuidar } \\
\text { dos idosos; na força de trabalho; na } \\
\text { promoção da inovação e na } \\
\text { pesquisa; e no atendimento } \\
\text { complexo aos idosos quanto as } \\
\text { necessidades de cuidados, por meio } \\
\text { do trabalho em parceria. }\end{array}$ & $\begin{array}{l}\text { Desenvolvimento de modelos de } \\
\text { serviços que combinem o ensino, a } \\
\text { aprendizagem e a pesquisa para } \\
\text { facilitar o atendimento aos idosos. }\end{array}$ & $\begin{array}{l}\text { Estudo descritivo com aporte da revisão de } \\
\text { documental e entrevistas com os participantes } \\
\text { interessados. O projeto CHART objetiva } \\
\text { facilitar o desenvolvimento de modelos de } \\
\text { serviços que combinem ensino, aprendizagem } \\
\text { e pesquisa para atendimento aos idosos Este } \\
\text { estudo descreve os processos e os } \\
\text { investimentos necessários para permitir que os } \\
\text { prestadores de cuidados façam parcerias com } \\
\text { as atividades de ensino e de pesquisa; com } \\
\text { estrutura e as práticas necessárias para o } \\
\text { desenvolvimento da capacidade da força de } \\
\text { trabalho e criação de planos de carreira no } \\
\text { setor. }\end{array}$ \\
\hline PC4 & $\begin{array}{l}\text { A educação interprofissional (EPI) é } \\
\text { essencial para preparar alunos de } \\
\text { enfermagem (PE) para prestar } \\
\text { cuidados em um ambiente de equipe } \\
\text { colaborativa. }\end{array}$ & $\begin{array}{l}\text { As percepções dos alunos sobre o } \\
\text { ambiente virtual de aprendizagem } \\
\text { que foi desenvolvido para melhorar } \\
\text { as competências interprofissionais. }\end{array}$ & $\begin{array}{l}\text { A coleta de dados foi realizada através de um } \\
\text { instrumento de pesquisa desenvolvido para } \\
\text { avaliar as competências da prática } \\
\text { interprofissional. Os participantes da pesquisa } \\
\text { foram os alunos }(\mathrm{n}=71) \text { partícipes de oito } \\
\text { disciplinas e oito discussões em } \\
\text { videoconferência sobre a apresentação de um } \\
\text { caso de paciente idoso em uma clínica virtual. }\end{array}$ \\
\hline PC5 & $\begin{array}{l}\text { Barreiras e caminhos para o } \\
\text { desenvolvimento, a manutenção de } \\
\text { uma agenda de pesquisa relevante } \\
\text { para as políticas de envelhecimento } \\
\text { em ambientes acadêmicos, bem como } \\
\text { os desafios adicionais associados à } \\
\text { participação em grupos minoritários } \\
\text { nessa busca. }\end{array}$ & $\begin{array}{l}\text { Desenvolvimento e manutenção da } \\
\text { agenda de pesquisa relevante para as } \\
\text { políticas de envelhecimento em } \\
\text { ambientes acadêmicos, e os desafios } \\
\text { adicionais associados à participação } \\
\text { em grupos minoritários. }\end{array}$ & $\begin{array}{l}\text { Estudo exploratório desenvolvido por meio de } \\
\text { grupo de focal }\end{array}$ \\
\hline РC6 & $\begin{array}{l}\text { Descrição dos papéis e das } \\
\text { competências essenciais para ANPs } \\
\text { que trabalham com idosos com } \\
\text { fragilidade para desenvolvimento de } \\
\text { um currículo. }\end{array}$ & $\begin{array}{l}\text { Desenvolvimento de um currículo } \\
\text { por meio dos papéis e das } \\
\text { competências essenciais para ANPs } \\
\text { que trabalham com idosos com } \\
\text { fragilidade. }\end{array}$ & $\begin{array}{l}\text { Revisão de literatura. Foram realizados } \\
\text { workshops com os representantes } \\
\text { multiprofissionais e leigos, o que gerou a } \\
\text { descrição de funções com uma lista de } 69 \\
\text { competências. Foi utilizado o processo Delphi } \\
\text { modificado com três rodadas envolvendo um } \\
\text { painel de } 31 \text { especialistas, incluindo } \\
\text { representantes do RCN, Comitê de Educação e } \\
\text { Treinamento da BGS, Grupo de Enfermeiros e } \\
\text { Praticantes Sênior da BGS, Rede da Sociedade } \\
\text { Chartered de Fisioterapia para Pessoas Idosas, } \\
\text { Colégio de Terapeutas Ocupacionais } \\
\text { Especialista em Pessoas Idosas Representantes } \\
\text { da seção e leigos. O consenso sobre as } \\
\text { declarações foi estabelecido por uma } \\
\text { concordância do painel de } 70 \% \text {. }\end{array}$ \\
\hline PC7 & $\begin{array}{l}\text { O programa de Aprendizagem } \\
\text { Facilitada para Avanço na Geriatria } \\
\text { (FLAG) foi projetado para aumentar } \\
\text { o número de professores de } \\
\text { enfermagem em programas de pré- } \\
\text { licenciamento com conhecimento } \\
\text { básico sobre envelhecimento e a } \\
\text { eficácia do ensino para preparar os } \\
\text { alunos para fornecer cuidados } \\
\text { seguros e de alta qualidade para } \\
\text { adultos mais velhos }\end{array}$ & $\begin{array}{l}\text { O Programa de Aprendizagem } \\
\text { Facilitada para Avanço na Geriatria } \\
\text { (FLAG) }\end{array}$ & $\begin{array}{l}\text { Uso da estrutura para melhorar a transferência de } \\
\text { aprendizagem, FLAG, este programa foi } \\
\text { projetado para incluir: (a) um workshop para } \\
\text { aumentar o conhecimento básico sobre } \\
\text { envelhecimento e síndromes geriátricas comuns, } \\
\text { e uso eficaz de estratégias de ensino / } \\
\text { aprendizagem baseadas em evidências; (b) um } \\
\text { programa de mentoria de um ano para apoiar a } \\
\text { aplicação do aprendizado em workshops e lidera } \\
\text { mudanças nas escolas dos participantes para } \\
\text { garantir que a geriatria seja uma prioridade. Os } \\
\text { métodos de avaliação formativa e somativa } \\
\text { foram usados e incluíram auto avaliação dos } \\
\text { objetivos, satisfação do programa e auto eficácia } \\
\text { de ensino. }\end{array}$ \\
\hline PC8 & $\begin{array}{l}\text { Uso do etnodrama como uma } \\
\text { intervenção para destacar o potencial } \\
\text { da velhice. }\end{array}$ & $\begin{array}{l}\text { Etnodrama utilizado para } \\
\text { intervenção com destaque do } \\
\text { potencial da velhice. }\end{array}$ & $\begin{array}{l}\text { Os participantes da pesquisa foram doze alunos } \\
\text { de bacharelado em enfermagem, os quais } \\
\text { constituíram pares com } 12 \text { residentes de uma } \\
\text { unidade de vida assistida para completar } \\
\text { atividades de aprendizagem transformadora }\end{array}$ \\
\hline
\end{tabular}




\begin{tabular}{|l|l|l|l|}
\hline & & $\begin{array}{l}\text { focadas no tópico do potencial da velhice. } \\
\text { Assim, culminou com o desenvolvimento de } \\
\text { um etnodrama a partir dos dados da pesquisa. } \\
\text { Foram realizadas transcrições dos conteúdos } \\
\text { das reuniões iniciais com os alunos; das } \\
\text { autorreflexões; do relato do desempenho dos } \\
\text { participantes da pesquisa; da discussão pós- } \\
\text { desempenho; e das questões abertas que foram } \\
\text { analisadas usando codificação. }\end{array}$ \\
\hline
\end{tabular}

Fonte: Levantamento de dados.

As contribuições estão na reflexão e discussão a respeito da formação profissional do enfermeiro na área da gerontologia, com a consulta às produções científicas para identificar o reconhecimento da enfermagem no âmbito do estudo.

Para a saúde, a relevância surge com a análise da formação sob o aspecto do cuidado do enfermeiro para o indivíduo, família e sociedade, da inclusão dos cuidadores familiares, bem como, a possibilidade de influenciar os processos de ensino e de aprendizagem para o desenvolvimento profissionais da área geriátrica para a qualidade deste cuidado. Há a probabilidade de verificação junto à comunidade acadêmica sobre as intervenções necessárias para o desenvolvimento da formação profissional do enfermeiro gerontológico, com métodos e estratégias adequadas a formação com matriz curricular que favoreça esta capacitação. Tal conhecimento poderá nortear a política de ensino nas IES, os grupos de pesquisas, os órgãos e as associações de classe que participam das discussões sobre a formação profissional do enfermeiro.

\section{Discussão}

A primeira produção científica PC1 traz a história curta e descritiva da prática do processo de aprendizagem, através do desenvolvimento de vinhetas de trabalho utilizadas como gatilho para instituir processos de aprendizagem e desenvolvimento profissional de enfermeiros geriátricos. A entrevista com 12 especialistas sobre o domínio da enfermagem geriátrica, oportunizou a compreensão de como as vinhetas devem ser projetadas e o seu aporte básico, através da observação do desenvolvimento de quatro histórias que descreviam as tarefas de trabalho da equipe de enfermagem geriátrica. Os resultados indicaram que o desenho de vinhetas baseado em critérios de escolha do estilo, conteúdo e a validação pode ser uma forma adequada de gerar possibilidades de aprendizado e desenvolvimento profissional (McCaffrey et. al., 2017).

Com o objetivo de ampliar a compreensão e os significados que os estudantes atribuíram ao novo exercício de leitura em relação ao seu desenvolvimento acadêmico, a PC2 revela que os romances são um recurso disponível aos educadores das disciplinas de saúde para apoiar a reflexão sobre a prática profissional e relações terapêuticas com os pacientes. Uma equipe interdisciplinar - incluindo enfermeiros, médico e instrutor de inglês - realizou um estudo interpretativo sobre o uso de um determinado romance por instrutores de enfermagem clínica em um curso de graduação. Esperava-se que os alunos colocados em unidades de convivência assistida e/ou de permanência de longo prazo para idosos lessem uma obra contemporânea, chamada Exit Lines de Joan Barfoot, onde a história é ambientada em um lar para idosos. A utilização da abordagem hermenêutica com o diálogo ao longo do estudo gerou perspectivas entre os participantes e a equipe de pesquisa interdisciplinar, possibilitando o surgimento da consciência dos alunos sobre a pluralidade epistemológica na enfermagem e a consequente importância de cultivar capacidade de reflexão sobre os diferentes pontos de vista e formas de saber (Jamieson \& Grealish, 2016).

Ao descrever os mecanismos de parceria (onze do setor de atenção residencial, ensino superior, setores hospitalares e não governamentais) que apoiaram o ensino e a pesquisa no cuidado ao idoso, em projetos de uma Cooperativa de Pesquisa e Ensino de Envelhecimento Saudável, a PC3 demonstrou que a manutenção do engajamento e a colaboração exigiu investimentos significativos e, muitas vezes invisíveis, em acordos de parceria que são constituídas em maior escala por 
pessoas, em vez de organizações dependentes. Através das ações desse grupo, novas colocações estudantis foram introduzidas e o apoio à continuidade das vagas de enfermagem permaneceu variável. A inovação da prática local avançou quando o investimento em parceria foi alinhado em níveis estratégicos e operacionais. O investimento contínuo e muitas vezes invisível na manutenção de parcerias operacionais tornou-se fundamental para a mudança sustentada. Em um ambiente privado de atendimento ao idoso a parceria foi viável para alcançar mudanças setoriais desafiadoras, tendo a inovação e melhoria de serviços como resultado do investimento (Palumbo, De Gagne \& Murphy, 2016).

Percebe-se que a educação interprofissional é essencial para tornar os profissionais de enfermagem capazes de prestar atendimento em um ambiente de trabalho com equipe colaborativa. A PC4, analisou a percepção dos alunos sobre o ambiente virtual de aprendizagem que foi desenvolvido para melhorar essas competências. Os instrumentos de pesquisa desenvolvidos serviram para avaliar alunos de oito disciplinas, após discussões em videoconferência sobre a apresentação de pacientes idosos em uma clínica virtual. Os participantes avaliaram positivamente a atividade, a prática da clínica virtual e videoconferência se tornaram uma plataforma de aprendizagem aceitável, podendo ser usada para simplificar a logística do ensino. Os papéis e o domínio da competência e responsabilidade é, talvez, o conceito mais difícil para os recém-formados entenderem. Essa metodologia de ensino apresentou-se como uma parte necessária para que os alunos possam usar essas habilidades no futuro (Kietzman et. al., 2016).

As mudanças no nível da política influenciam de maneira significativa na saúde e no bem-estar das populações envelhecidas. No entanto, é comum existir um abismo entre o conhecimento científico e a ação política. Embora, pesquisas anteriores tenham identificado barreiras e ações facilitadoras para a tradução efetiva do conhecimento, pouca atenção tem sido dada ao papel das instituições acadêmicas na geração do conhecimento. A PC5 apresentou um estudo exploratório com de pesquisadores que examinaram as barreiras e caminhos para o desenvolvimento e a manutenção de uma agenda de pesquisa relevante para a política de envelhecimento em ambientes acadêmicos, além dos desafios adicionais associados à adesão de grupos minoritários nessa busca. Segundo os autores, os participantes do recorte encontravam-se pessoalmente comprometidos com a realização de pesquisas relevantes para as políticas, apesar de surgir barreiras institucionais, como menos oportunidades de financiamento e menos valor atribuído à pesquisa, particularmente no contexto de posse e promoção. Embora muitos enxergassem a pesquisa como uma oportunidade de fazer a diferença - especialmente para populações de idosos mais carentes -, vários sujeitos disseram que seus interesses políticos de pesquisa eram marginalizados. Dentro e fora das instituições acadêmicas, eles ofereceram estratégias individuais e institucionais para superar as dificuldades, colaborando mutuamente com os membros da comunidade, colegas e mentores. A reformulação da valorização da pesquisa por meio da diversificação de oportunidades de financiamento e publicação pode apoiar estudos sobre o envelhecimento, que são relevantes para as políticas públicas (Goldberg et. al., 2016).

Os profissionais de enfermagem avançada são enfermeiros experientes, que realizam algumas atividades que são tradicionalmente realizadas pela equipe médica. Nesse campo, existem quatro pilares de habilidades: clínicas avançadas, liderança, educação e pesquisa. Esses profissionais começaram a se especializar no atendimento à população idosa, por isso, $\mathrm{o}$ papel e as competências necessárias não foram bem definidos. A PC6 estabeleceu um consenso especializado sobre a descrição da função e competências essenciais para o desenvolvimento do currículo para enfermeiros que trabalham com idosos. O resultado trouxe a descrição do papel do enfermeiro em três rodadas: a primeira, acordou vinte e cinco competências essenciais, aumentando para 43 na segunda rodada e 49 após a terceira. Nesse cenário, o estudo de Delphi permitiu a elaboração de um painel nacional de especialistas clínicos e representantes leigos para refinar e concordar com um conjunto de competências para que o enfermeiro possa cuidar de pessoas mais velhas com fragilidade. É o primeiro passo para garantir a consistência na formação do enfermeiro gerontológico (Krichbaum et. al., 2015). 
A elaboração do Programa de Aprendizagem Facilitada para Avançar na Geriatria serviu para aumentar o número de professores de enfermagem com conhecimentos básicos sobre envelhecimento e efetividade de ensino, para a preparação de alunos que prestem cuidados seguros e de alta qualidade aos idosos. A PC7 identificou a utilização da estrutura do programa para melhorar a transferência de aprendizagem, sendo o mesmo projetado para oferecer: oficina para aumentar o conhecimento básico sobre o envelhecimento e as síndromes geriátricas comuns, assim como o uso efetivo de estratégias de ensino/aprendizagem baseadas em evidências; e, programa de mentoria para apoiar a aplicação da aprendizagem em oficinas e a mudança de liderança nos cursos dos participantes, para garantir que a geriatria seja uma prioridade (Eaton, 2015).

Como métodos de avaliação formativa e sumária, foram utilizados a auto avaliação dos objetivos, satisfação do programa e auto eficácia do ensino de 152 participantes de 19 estados, incluindo 23 professores de programas de graduação associados e 102 de programas de bacharelado. A eficácia do ensino auto avaliado melhorou significativamente de pré para pós-oficina a cada ano. O alcance dos objetivos de aprendizagem foi avaliado com satisfação e a transferência da aprendizagem foi evidenciada pela implantação de projetos educacionais em escolas apoiadas pela mentoria. O programa proporcionou oportunidades para os educadores de enfermagem aprenderem a ensinar geriatria de forma mais eficaz e a transferir o aprendizado para o seu ambiente de trabalho. Os programas futuros serão oferecidos em formato encurtado, incorporando conteúdos e estratégias online, agregando ao público outros profissionais de saúde, com o mesmo objetivo de aumentar os conhecimentos e habilidades dos educadores para preparar os alunos para prestar cuidados com excelência aos idosos (Eaton, 2015).

A transposição de barreiras para a expansão da formação dos prestadores de cuidados de saúde geriátricos é o desejo dos estudantes de enfermagem que almejam trabalhar com idosos. A PC8 avaliou a viabilidade do uso do etnodrama como intervenção para destacar o potencial da vida tardia. Na ocasião, doze estudantes de bacharelado em enfermagem foram direcionados a centro de convivência assistido junto com outros doze residentes para a realização de atividades de aprendizagem focadas no tema do potencial de vida tardia, culminando na realização de um etnodrama desenvolvido a partir desses dados. Foram analisadas transcrições de reuniões iniciais, autorreflexões, desempenho, discussão pós-desempenho e perguntas abertas da pesquisa por meio de codificação in vivo e padrão. Enquanto os participantes idosos reconheceram e enfatizaram o potencial positivo de vida tardia, os alunos exploraram o potencial humano ao longo da vida e refletiram sobre o que isso significa em suas próprias vidas. Percebe-se que a constante discussão sobre o potencial da vida tardia pode alterar o estigma associado ao envelhecimento (Cherman \& Rocha-Pinto, 2016).

O conhecimento, a avaliação crítica e a síntese das evidências existentes neste estudo estão ancoradas em Bardin (2011), quando sugere que a interpretação das falas dos sujeitos se concretiza através do sentido que eles atribuem às mensagens, mediante a classificação dos elementos constitutivos de um todo, por diferenciação e, consequentemente, por reagrupamento direcionado pelo gênero, isto é, através da analogia que possui critérios previamente definidos.

Não apenas a própria enfermagem está relacionada com a extensão de resultados significativos da formação profissional, estando também, entrelaçada com o processo de cuidar da pessoa idosa e a qualidade do cuidado familiar. Neste sentido, a formação profissional do enfermeiro na área da gerontologia vem sendo fortalecida pelos métodos e estratégias utilizadas nos processos de ensino e de aprendizagem para o desenvolvimento profissional dos enfermeiros, principalmente quando Hildegard Peplau (2014) instituiu a visão singular para compreender a afinidade enfermeiro-paciente no campo da enfermagem, ao ponderar as áreas da prática clínica, da teoria e da pesquisa, por se expandir a base de conhecimento para a Enfermagem.

A formação profissional do enfermeiro na área da gerontologia é reconhecida pelo cuidado de enfermagem à família, o que fortalece os processos de aprendizagem e desenvolvimento profissional dos enfermeiros. $\mathrm{O}$ ensino e a pesquisa sobre $\mathrm{o}$ cuidado ao idoso demonstra que a manutenção do engajamento e a colaboração do enfermeiro exigiram investimentos 
significativos para equipe de enfermagem. No entanto, as práticas avançadas se apresentaram nesta revisão como pilares das habilidades clínicas, juntamente com os temas: liderança, educação e pesquisa. Os profissionais de enfermagem avançada realizam atividades que são tradicionalmente realizadas pela equipe médica e neste estudo tais práticas se destacaram.

Outro tema que emerge desta revisão é a necessidade de a enfermagem gerontológica brasileira contextualizar o cuidado segundo a cultura brasileira, que inclui diferenças regionais importantes. Vale ressaltar, que, qualquer tentativa de homogeneização cultural através da educação precisa ser denunciada, criticada para que não se imponha um "discurso" ideológico que se incorpore ao pedagógico. A formação discursiva torna-se o lugar da constituição do sentido e da identificação do sujeito. É nela que todo sujeito se reconhece - em sua relação consigo mesmo e com os outros -, fazendo surgir a condição do consenso intersubjetivo, que é a evidência de $e u$ e $t u$, onde o sujeito se identifica e adquire identidade.

Nesse sentido, o educador precisa estar atento à possibilidade de imposição da cultura das classes dominantes. Quando a instituição de ensino impõe uma cultura particular das classes dominantes, nas máscaras de sua natureza social e cultural apresentadas como objetivo indiscutível, rejeitando as culturas de outros grupos sociais, legitima a arbitrariedade cultural (Boudieu \& Passeron, 1992).

Sendo assim, a violência simbólica como produto da imposição de uma cultura arbitrária, por meio de atores, práticas e instituições também, arbitrariamente selecionados e destinados a tal função, precisa ser identificada e discutida. Tal violência não pode ser esquecida, pois uma vez que atravessa todo o seio social, forma consciências e condiciona práticas sociais, é naturalizada e cumpre a missão de reproduzir a ordem social dominante, de maneira dissimulada e socialmente aceita (Boudieu \& Passeron, 1992).

\section{Considerações Finais}

Segundo a pesquisa, as estratégias de ensino utilizados na educação em enfermagem geriátrica foram: 1. Vinhetas por meio da observação do desenvolvimento de histórias; 2. Uso de romances de abordagem hermenêutica com o diálogo; 3. Pesquisa no cuidado ao idoso em projetos; 4. Educação interprofissional; 5. Ambiente virtual de aprendizagem - vídeo conferência; 6. Estudo exploratório; 7. Programa de Aprendizagem Facilitada; 8. Método Delphi; 9. Oficinas; 10. Programa de mentoria; 11. Programa baseado em evidências; e 12. Etnodrama. Sendo os tipos de avaliação mais utilizados, auto avaliação e formativa.

Dentre os temas estudados, os mais encontrados foram: educação em enfermagem; enfermagem geriátrica/educação; geriatria/educação; materiais de ensino; ensino; competência clínica; educação; educação em enfermagem/métodos; educação em enfermagem/normas; humanos; motivação; melhoria de qualidade. Sendo assim, torna-se relevante a pesquisa sobre a formação do enfermeiro em gerontologia e como ela se constitui nos cursos de graduação no Brasil e no exterior.

Por meio da análise das produções científicas, verificou-se o reconhecimento do enfermeiro e sua formação profissional na área da gerontologia, sendo a sua formação fortalecida por métodos e estratégias inovadores, utilizados na constituição dos processos de ensino e de aprendizagem para o desenvolvimento profissional. Aí se revela a importância da ciência da enfermagem e dos relacionamentos interpessoais.

A formação profissional do enfermeiro é evidenciada por meio da prática avançada e das atividades que são tradicionalmente realizadas pela equipe médica através de certas habilidades de clínicas avançadas (liderança, educação e pesquisa). Portanto, existe a necessidade de definição do "papel", da função e das competências do enfermeiro, com a reflexão crítica sobre a relação da teoria dos bens simbólicos, tendo em vista que a autonomia é relativa ao campo cultural e das relações de classes. A escola nasce como principal instância de legitimação da desigualdade social, com a contribuição da reprodução da estrutura de distribuição de capital cultural entre as classes e sua transmissão. 
Entende-se ser imperiosa a consignação do consenso especializado sobre a descrição da função e das competências do enfermeiro gerontológico. Elas são essenciais para o desenvolvimento, formação e definição do perfil profissional, e do currículo para quem trabalha com idosos. Apesar disso, a reflexão crítica deve se fazer presente, para que a educação seja constituída por um trabalho contínuo de revelação, de análise e de crítica ao "discurso" ideológico, a fim de valorizar-se a incorporação de um "discurso" pedagógico.

Percebe-se a existência de inovação da prática local avançada, principalmente, quando se faz investimento em parceria alinhada com a proposta de ensino (em níveis estratégicos e operacionais), bem como investimento contínuo. Até mesmo porque é comum não perceber este investimento na manutenção de parcerias operacionais, porquanto é básico para a mudança sustentável.

Considera-se importante a elaboração de um painel nacional de especialistas clínicos e representantes leigos, com o intuito de refinar e estrutura um conjunto de competências necessários para o enfermeiro, no que se refere ao cuidado de pessoas idosas com fragilidade. Sendo essa ação, o primeiro passo para garantir a consistência na formação do enfermeiro gerontológico. Mas, verifica-se nesse ponto, que é preciso ter um olhar atento para o contexto social em que o profissional está inserido, para evitar a alienação cultural e a imposição da cultura das classes dominantes.

As produções científicas reconhecem o cuidado de enfermagem na família e aos cuidadores familiares, por meio dos processos de aprendizagem e desenvolvimento profissional de enfermeiros geriátricos, possibilitando a qualidade deste cuidado. Conclui-se que as estratégias para os processos de aprendizagem descritos no estudo oportunizam a compreensão das tarefas de trabalho da equipe de enfermagem geriátrica; geram possibilidades de aprendizado e desenvolvimento profissional e; apoiam a reflexão sobre a prática profissional e relações terapêuticas com os pacientes.

Os estudos apontam haver apoio ao ensino e pesquisa no cuidado ao idoso, o que demonstrou engajamento e investimentos significativos das instituições de ensino, o que garantiu a inovação da prática local. O investimento esteve alinhado com a proposta de ensino em níveis estratégicos e operacionais. Tal investimento contínuo e muitas vezes invisível na manutenção de parcerias operacionais é fundamental para a mudança sustentada. Assim, o financiamento para a educação se torna um fator importante.

Considera-se, finalmente, que não apenas a própria enfermagem está relacionada com a extensão de resultados significativos da formação profissional, ela também está entrelaçada com a qualidade do cuidado à pessoa idosa e sua família. Sendo assim, sugere-se a realização de estudos futuros com as temáticas voltadas para o cuidado da pessoa idosa com vistas a qualidade da assistência; a formação do enfermeiro para o atendimento das diferentes etapas de vida, inclusive para o envelhecimento saudável; e os fatores que interferem na formação do enfermeiro para o alcance da competência no cuidado ao idoso.

\section{Referências}

Almeida, V. C. F, Lopes, M. V. O. \& Damasceno, M. M. C. (2005). Teoria das relações interpessoais de Peplau: análise fundamentada em Barnaum. Revista da Escola de Enfermagem, 39(2), 202-210.

Babini, M. (2006). Do conceito à palavra: os dicionários onomasiológicos. Ciência e Cultura, 58(2), 38-41.

Bardin, L. (2011). Análise de conteúdo. Edições 70.

Boudieu, P. F. \& Passeron, J. C. (1992). A Reprodução: elementos para uma teoria do sistema de ensino. (3.ed.) Rio de Janeiro: Francisco Alves.

Bourdieu, P. (2007). A economia das trocas simbólicas. Perspectiva.

Brasil. (1988). Constituição da República Federativa do Brasil. Capítulo VII. Art 230. Da Família, da Criança, do Adolescente e do Idoso. <http://www.planalto.gov.br/ccivil_03/constituicao/constituicao.htm>.

Brasil. (1990). Casa Civil. Lei $n^{\circ}$ 8.080, de 19 de setembro de 1990. Dispõe sobre as condições para a promoção, proteção e recuperação da saúde, a organização e o funcionamento dos serviços correspondentes e dá outras providências. < http://www.planalto.gov.br/ccivil_03/leis/18080.htm>. 
Research, Society and Development, v. 10, n. 11, e426101119813, 2021

(CC BY 4.0) | ISSN 2525-3409 | DOI: http://dx.doi.org/10.33448/rsd-v10i11.19813

Brasil. (2006). Ministério da Saúde. Portaria $n^{o}$ 2.528, de 19 de outubro de 2006. Aprova a Política Nacional de Saúde da Pessoa Idosa. <https://bvsms.saude.gov.br/bvs/saudelegis/gm/2006/prt2528_19_10_2006.html>.

Cherman, A. \& Rocha-Pinto, S. R. (2016). Fenomenografia e valoração do conhecimento nas organizações: diálogo entre método e fenômeno. Revista De Administração Contemporânea, 20(5), 630-650.

DeCS (2016). Descritores em Ciências da Saúde. <https://decs.bvsalud.org/P/decs2016p.htm>.

Eaton, J. (2015). The feasibility of ethnodrama as intervention to highlight late-life potential for nursing students and older adults. Gerontology \& Geriatrics Education, 36(2), 204-22.

Goldberg, S. E. et. al. (2016). Development of a curriculum for advanced nurse practitioners working with older people with frailty in the acute hospital through a modified Delphi process. Age Ageing, 45(1), 48-53.

IBGE. (2018). Instituto Brasileiro de Geografia e Estatística. Projeções da população: Brasil e unidades da federação. IBGE. <https://biblioteca.ibge.gov.br/index.php/biblioteca-catalogo?view=detalhes\&id=2101597>.

Jamieson, M. \& Grealish, L. (2016). Co-operative working in aged care: The Cooperative for Healthy Ageing Research and Teaching Project. Australasian Journal on Ageing, 35(3), 22-28.

Kietzman, K. G. et. al. (2016). Pathways to Advancing Aging Policy-Relevant Research in Academic Settings. Gerontology \& Geriatrics Education, 37(1), 81-102.

Krichbaum, K. et. al. (2015). Facilitated Learning to Advance Geriatrics: Increasing the Capacity of Nurse Faculty to Teach Students About Caring for Older Adults. The Gerontologist, 55, 154-164.

Leicher, V. \& Mulder, R. H. (2018). Development of vignettes for learning and professional development. Gerontology \& Geriatrics Education, 39(4), 464480.

Marton, F. \& Booth, S. A.(1997). Learning and awereness. Lawrence Erlbaum Inc. Publishers.

Marton, F. (1981). Phenomenography - describing conceptions of the world around us. Instructional Science, 10(2), 177-200.

McCaffrey, G. et. al. (2017). Bringing a novel to practice: An interpretive study of reading a novel in an undergraduate nursing practicum course. Nurse Education in Practice, 24, 84-89.

Mendes, K. D. S, Silveira, R. C. C. P. \& Galvão, C. M. (2008). Revisão integrativa: método de pesquisa para a incorporação de evidências na saúde e na enfermagem. Texto \& Contexto Enfermagem, 17(4), 758-764.

Palumbo, M. V, De Gagne, J. C. \& Murphy, G. (2016). Interprofessional care of elders: Utilizing the virtual learning environment. Journal of the American Association of Nurse Practitioners, 28(9), 465-70.

Peplau, H. E. (2014). Interpersonal relations in nursing: A conceptual frame of reference for psychodynamic nursing. Putnam. In: Chrizostimo, M.M. El Desafío de la Formación Profesional del Enfermero con Compromiso Social (Tesis de Doctorado). Argentina: Universidad Nacional de Rosario.

Sandberg, J. (2000). Understanding human competence at work: an interpretative approach. Academy of Management Journal, 43(1), 9-25. 